\title{
Moderate Hallucination
}

National Cancer Institute

\section{Source}

National Cancer Institute. Moderate Hallucination. NCI Thesaurus. Code C156243.

Occasional verbal, visual, gustatory, olfactory or tactile hallucinations with no functional impairment; or non-verbal auditory hallucinations/visual illusions more than infrequently or with impairment. 